\title{
CAVITATION \\ REACTION ENGINEERING
}




\section{The Plenum Chemical Engineering Series}

Series Editor:

Dan Luss, University of Houston, Houston, Texas

\section{CAVITATION REACTION ENGINEERING}

Y. T. Shah, A. B. Pandit, and V. S. Moholkar

COAL COMBUSTION AND GASIFICATION

L. Douglas Smoot and Philip J. Smith

ENGINEERING FLOW AND HEAT EXCHANGE, Revised Edition Octave Levenspiel

INDUSTRIAL CRYSTALLIZATION: Process Simulation Analysis and Design Narayan S. Tavare

REACTION ENGINEERING OF STEP GROWTH POLYMERIZATION

Santosh K. Gupta and Anil Kumar

THE STRUCTURE AND REACTION PROCESSES OF COAL

K. Lee Smith, L. Douglas Smoot, Thomas H. Fletcher, and Ronald J. Pugmire

TRANSPORT MECHANISMS IN MEMBRANE SEPARATION PROCESSES

J. G. A. Bitter

A Continuation Order Plan is available for this series. A continuation order will bring delivery of each new volume immediately upon publication. Volumes are billed only upon actual shipment. For further information please contact the publisher. 


\section{CAVITATION \\ REACTION \\ ENGINEERING}

Y. T. Shah

Clemson University

Clemson, South Carolina

and

A. B. Pandit and V. S. Moholkar

University of Bombay

Bombay, India

Springer Science + Business Media, LLC 


\section{Library of Congress Cataloging-in-Publication Data}

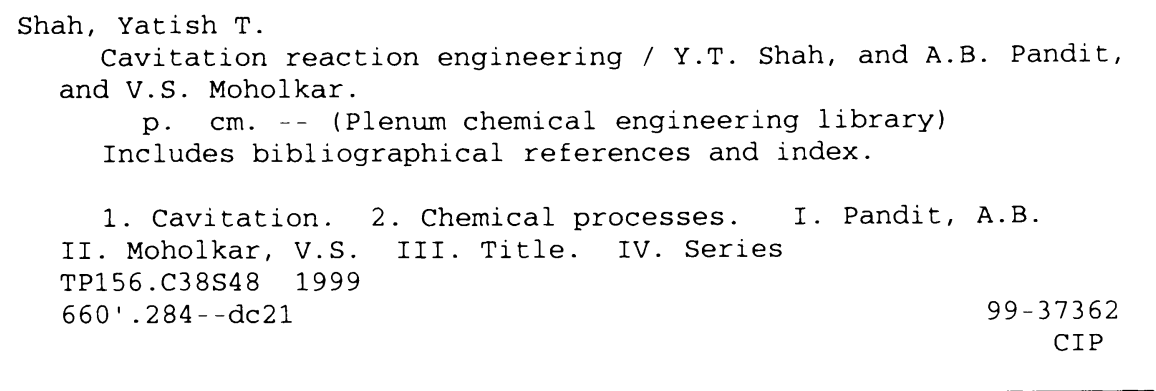

ISBN 978-1-4613-7168-7

ISBN 978-1-4615-4787-7 (eBook)

DOI 10.1007/978-1-4615-4787-7

(C) 1999 Springer Science+Business Media New York

Originally published by Kluwer Academic / Plenum Publishers in 1999

Softcover reprint of the hardcover 1st edition 1999

$\begin{array}{llllllllll}10 & 9 & 8 & 7 & 6 & 5 & 4 & 3 & 2 & 1\end{array}$

A C.I.P. record for this book is available from the Library of Congress.

All rights reserved

No part of this book may be reproduced, stored in a retrieval system, or transmitted in any form or by any means, electronic, mechanical, photocopying, microfilming, recording, or otherwise, without written permission from the Publisher 
To James, Keith, Jonathan, and Heather Y.T.S. 


\section{PREFACE}

The literature on cavitation chemistry is ripe with conjectures, possibilities, heuristic arguments, and intelligent guesses. The chemical effects of cavitation have been explained by means of many theories, consisting of empirical constants, adjustable parameters, and the like. The chemists working with cavitation chemistry agree that the phenomenon is very complex and system specific. Mathematicians and physicists have offered partial solutions to the observed phenomena on the basis of cavitation parameters, whereas chemists have attempted explanations based on the modes of reaction and the detection of intermediate chemical species. Nevertheless, no one has been able to formulate a unified theme, however crude, for its effects on the basis of the known parameters, such as cavitation and transient chemistry involving extremely high temperatures of nanosecond durations.

When one surveys the literature on cavitation-assisted reactions, it is clear that the approach so far has been "Edisonian" in nature. While a large number of reactions have showed either enhanced yields or reduced reaction times, many reactions have remained unaffected in the presence of cavitation. The success or failure of cavitation reactions ultimately depends on the collapse of the cavity.

Cavitation chemistry is based on the principles of the formation of small transient cavities, their growth and implosion, which produce chemical reactions caused by the generation of extreme pressures and temperatures and a high degree of microturbulence. Cavities can be generated either by expansion of pressure (caused hydrodynamically or acoustically) or by sharp penetration of energy by lasers or protons. This monograph deals with the reaction engineering associated with the cavitation phenomenon and its practical applications in the treatment of various types of aqueous waste or for organic synthesis.

Mathematical modeling of cavitation phenomena and the related chemical effects is rather complex; even for the simplest reactions, there appears no possibility of a generalized theory in the near future. The chemical effects observed and reported in the literature as either transient or time averaged most definitely have resulted from the cavitation phenomenon. All researchers and a few practitioners will not 
have any difficulty in accepting newly coined terms of cavitation chemistry and cavitation reaction engineering, which encompasses all aspects of the chemical transformations caused by the cavitation phenomenon.

This monograph is an attempt to present the observed effects on a common basis, namely, the parameters affecting cavitation, those affecting chemistry, and those affecting both, which are important in an appropriate design of a cavitation reactor. The mathematical modeling of cavitation bubble dynamics, chemical reactions, and reactors is discussed, with an emphasis on the physical explanations of the phenomena on the basis of known parameters affecting cavitation or parameters affecting the chemical transformations and the performance of the reactors.

The cavitation process can be categorized according to the method used to produce cavities. Hydrodynamic cavitation is produced by the pressure variation in a flowing liquid that is caused by the variation of velocity in the system. At the points of highest velocity and lowest pressure, cavities can occur. These cavities can (1) move with the liquid and subsequently collapse (i.e., traveling cavitation); (2) remain fixed at a rigid surface under unsteady state condition (i.e., fixed cavitation); or (3) reside in the cores of vortices (i.e., vortex cavitation) that form in the regions of high shear and boundary layer separation. Traveling cavitation is most efficient for chemical conversion. Acoustic cavitation is produced by sound waves in a liquid that are caused by pressure variations. Optical cavitation is produced by a laser light rupturing the liquid. Finally, particle cavitation is produced by the deposition of energy using any other type of elementary particle, such as a proton.

Chapter 1 briefly discusses the underlying physics behind the sources of cavitation. Instruments are currently available to produce cavities from these different sources. Cavitation bubbles and their behavior under different environments form the basis for how the cavitation process affects chemical transformation.

A suitable mathematical description of bubble dynamics and their interaction with the surrounding liquid medium is given in Chapter 2. While a complete description of bubble behavior is rather complex, the mathematics outlined in this chapter form the basis upon which more complex models can be developed. The modifications of the equations and the incorporation of the parameters involving physical effects are presented for different modes of cavitation. Different types of cavitation are discussed on the basis of the mode of energy dissipation and the time scales involved for this energy dissipation. The similarities and differences arising are discussed as part of a unified theme of energy dissipation and conversion for cavitation.

Finally, the physical parameters that affect the nature of the process in each type of cavitation are described in Chapter 3. The relationship between the physical parameters and the nature of the cavitation behavior described in this chapter is qualitatively and in some instances quantitatively obtained from an evaluation of the basic equations for cavitation bubble dynamics. 
The success or failure of cavitation-assisted reactions ultimately depends on the active or reactive species generated as a result of cavity collapse. The cavitation phenomenon consists of three stages: nucleation, growth, and collapse of a single cavity or a cluster of cavities. A scientific approach to cavitation chemistry requires the control of these three stages.

Although nucleation is a fairly random phenomenon, considerable control over the rate has been achieved in the crystallization process by controlling the degree of supersaturation (metastable region) or by the addition of external nuclei (seeding). It may be possible to do the same for the nucleation of cavities or a cluster of cavities. Growth and collapse behavior of the cavity or the cluster of cavities is controlled by the surrounding fluctuating pressure field, i.e., its frequency and amplitude. Since the generation of active and reactive species depends on this cavity behavior, the success or failure of the reaction is related to the frequency and amplitude of the surrounding pressure field.

It is expected that from Chapters $1-3$, the reader will be made aware of the common underlying theme responsible for a variety of chemical effects reported in the literature. The reader will also become conversant with cavitation as a physical phenomenon and the factors affecting this physical phenomenon. Where there is no literature on an experimental proof of the physical phenomena predicted by the mathematical and physical equations, numerical predictions have been used liberally to explain cavitation-related physical effects. This involves the behavior of a single cavity or a cluster of cavities in a variety of surroundings; i.e. form, mode, and time scales of energy dissipation.

Cavitation can have beneficial effects on a reaction as a result of physical effects such as microstreaming or chemical effects such as generation of $\mathrm{OH}^{-}$radicals (hydrolysis or oxidative reactions). Control of these effects requires a fundamental analysis of cavity dynamics and not a simple use of cavitation intensity, as is often done in the literature. In Chapters 4 and 5 on gas-liquid cavitation chemistry and gas-liquid-solid cavitation chemistry, an attempt has been made to relate or use the predicted and observed physical effects of cavitation to explain the chemical and physical transformations observed and reported in the literature. Wherever possible, the parameters responsible for the chemical transformations and the changes in these parameters as a result of the physical phenomena of cavitation are highlighted. Heterogeneous cavitation chemistry and homogeneous cavitation chemistry, which are incorrectly separated in the literature, are presented on a common footing here because cavitation chemistry is always heterogeneous (more than one phase) owing to the formation of vapor- or gas-filled cavities. The correct separation is indicated in the titles of these two chapters.

Various possible and suggested mechanisms of cavitation-aided practical chemical reactions are also briefly discussed in Chapters 4 and 5 . The interesting behavior of a variety of chemical reactions in the presence of cavitation is illustrated with the support of the experimental facts. The emphasis is largely on chemical trans- 
formations in the aqueous phase so that the results are useful in treating aqueous waste. The mode of generation of the cavitation, its physical effect, and its ultimate utilization in chemical conversion is discussed in detail, linking the physical effects of cavitation and the followup chemical transformations.

The next three chapters (Chapters 6,7, and 8) deal with the engineering translation of the basic physical and chemical concepts discussed in Chapters 1 to 5 into their possible practical uses. Various designs reported in the literature using hydrodynamic, acoustic, or laser energy for the generation of cavitation are discussed in terms of operational and design difficulties and ease of the chemical transformation. New, probable, and/or novel reactor configurations have also been proposed. Specific examples have been selected to highlight the difficulty or the ease of operation of various types of cavitational reactors. Examples have been used with the results to discuss the efficacy of one type of cavitation compared with another. The effect of physicochemical properties and the optimum use of cavitation for the required chemical effects are also discussed.

In these chapters, the problems associated with energy efficiencies and scaleup are also discussed. It has been found that the industrial success of cavitation reactors and their design and optimization strategies differ from system to system or are system specific. The optimization of the cavitation reactor as required for any one system is not necessarily applicable to other systems. The specific parameters responsible for the success of this optimization are highlighted. Some new reactors for specific applications are proposed. These chapters are expected to generate curiosity and research interest in the people working in the field of cavitation chemistry and cavitation reactors.

Chapter 7 deals with mathematical models for cavitation reactors. This subject is very difficult because of the lack of appropriate experimental data, the complexity of the system, and the difficulty in predicting a reactor's behavior at different scales. Numerous models based on different sets of assumptions for the reaction zone are described. Probability density function is used to illustrate the behavior of the reaction zone in an acoustic cavitation reactor.

The cost effectiveness of a cavitation reactor depends very strongly upon the energy efficiency of the steps leading to the chemical transformation. The energy efficiency of the cavity implosion process as a function of frequency is discussed in Chapter 8. The range of frequency covers all types of processes and reactors, such as hydrodynamic (low frequency), acoustic (medium frequency), and laser (high frequency) cavitation. The performance of the acoustic reactor is also evaluated based on the concept of sonochemical yield, an empirical parameter that can help in comparing and evaluating the performance of various types of acoustic reactors.

Chapter 8 also uses concepts discussed in earlier chapters for the design and optimization of a cavitation reactor. It briefly presents some economic comparisons of a few standard effluent treatment and recovery techniques with new techniques 
of cavitation-based chemical conversions. Both capital and the operating costs of conventional and nonconventional hydrodynamic, acoustic, and other types of cavitation reactors are briefly discussed. The need for further research is delineated. The chapter first deals with the efficiency of the energy conversion supplied for the global cavitational effect and then the optimized cavitational effect, i.e., the one required for a specific chemical or physical conversion. The results are illustrated with case studies.

Finally, Chapter 9 describes some pilot and large-scale applications of the cavitational chemical transformations. The examples clearly illustrate the commercial viability of the concept discussed in this monograph. While the examples are largely for a hydrodynamic cavitation process because of its favorable economics, a similar development for acoustic cavitation should appear in the near future. Commercial applications for laser and particle cavitations may require more development to make these technologies more economical.

Cavitation reaction engineering is rapidly changing, largely because of its ease of application to a variety of chemical reactions. It is hoped that this monograph summarizes the state of knowledge available on this subject and will fuel more momentum for research in this area. It is our hope that with increased knowledge of the subject, the present "Edisonian" approach will be replaced by a more scientific assessment of the possibilites and applications of cavitation processes.

A monograph such as this would not have been possible without the support of many people. We would like to thank Profs. Luss, Doraiswamy, Sharma, and Joshi for their constant encouragement on this project. One of the authors (ABP) would like to acknowledge the University Grants Commission and the Department of Science and Technology, Government of India for giving him the opportunity to work in this exciting field. We would also like to thank our students, in particular Parag Gogate, Nilesh Vichare and Prashant Tatake for their assistance and comments on the manuscript. Our sincere thanks to Mrs. Melody Land for all the hard and painstaking detail work she carried out for the completion of this project. Our thanks also to Mr. Ron Baker for his help with the drawings. Finally, and most importantly, projects such as this cannot be completed without the love and support of our wives, Mary and Anala, and our children. 


\section{CONTENTS}

\section{Sources and Types of Cavitation}

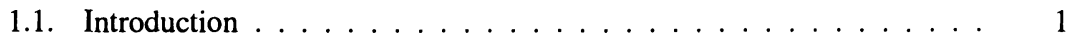

1.2. Hydrodynamic Cavitation . . . . . . . . . . . . . . . . 3

1.2a. Cavitation Number . . . . . . . . . . . . . . . . 4

1.3. Acoustic Cavitation . . . . . . . . . . . . . . . . 8

1.4. Optic and Particle Cavitation . . . . . . . . . . . . . . . . . 14

\section{Cavitation Bubble Dynamics}

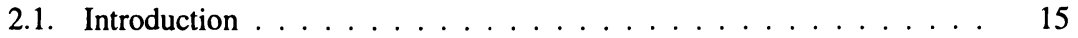

2.2. Bubble Dynamics . . . . . . . . . . . . . . . . . . . 16

2.2a. Bubble Nuclei: Blake Threshold . . . . . . . . . . . . . . 17

2.2b. Dynamic Equations of a Spherical Bubble: Analysis of an Empty Bubble . . . . . . . . . . . . . . . . 20

2.2c. Dynamics of a Gas Bubble . . . . . . . . . . . . . . 21

2.2d. Equation Involving Compressibility of a Liquid . . . . . . . . . 24

2.2e. Rayleigh Analysis of a Cavity and Its Extensions . . . . . . . . . 26

2.2f. Adiabatic Collapse of a Gas-Filled Cavity . . . . . . . . . 28

2.2g. Damping of Stable Bubbles . . . . . . . . . . . . . 30

2.2h. Modifications for Hydrodynamic Cavitation . . . . . . . . 33

2.3. Cluster Dynamics . . . . . . . . . . . . . . . . . . . . . 36

2.3a. Model Equations for Cluster Dynamics . . . . . . . . . . . . . 37

2.4. Heat and Mass Transfer Effects in Cavitation . . . . . . . . . . . . . 39

2.4a. Rectified Diffusion . . . . . . . . . . . . . . . . . 39

2.4b. Rectified Heat Transfer in Bubble Oscillations . . . . . . . . . . 47

2.4c. Effect of Simultaneous Diffusion and Evaporation on

Bubble Dynamics . . . . . . . . . . . . . . . . . 50

2.5. Concluding Remarks ... . . . . . . . . . . . . . . . 54 


\section{Factors Affecting Cavitation Behavior}

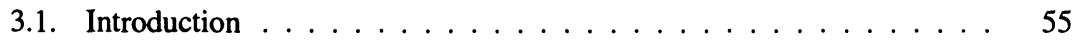

3.2. Factors Affecting Cavity Behavior in Hydrodynamic Cavitation . . . . . 55

3.2a. Recovered Discharge Pressure and Time of Pressure Recovery . . 57

3.2b. Downstream Pipe Size . . . . . . . . . . . . . . . . . . . 62

3.2c. Orifice-to-Pipe Diameter Ratio . . . . . . . . . . . . . . . . 62

3.2d. Initial Bubble Radius and the Noncondensable Gas Fraction in

Cavitating Liquids . . . . . . . . . . . . . . . . . 62

3.3. Factors Affecting Cavity Behavior in Acoustic Cavitation . . . . . . . 66

3.3a. Acoustic Frequency . . . . . . . . . . . . . . . . 66

3.3b. Acoustic Intensity . . . . . . . . . . . . . . . 72

3.3c. External Pressure . . . . . . . . . . . . . . . . . . 72

3.3d. Nature of the Dissolved Gas . . . . . . . . . . . . . . . 73

3.3e. Physical Properties of the Cavitating Medium . . . . . . . . . . 74

3.3f. Pretreatment of the Liquid . . . . . . . . . . . . . . . . 74

3.3g. Bulk Liquid Temperature . . . . . . . . . . . . . . . . 74

3.3h. Initial Bubble Radius . . . . . . . . . . . . . . . . . . . 74

3.4. Factors Affecting Optical Cavitation . . . . . . . . . . . . . 75

3.5. Factors Affecting Cavity Cluster Behavior in Hydrodynamic

Cavitation . . . . . . . . . . . . . . . . . . 77

3.5a. Effect of Recovery Pressure . . . . . . . . . . . . . . . 78

3.5b. Effect of Time of Pressure Recovery . . . . . . . . . . . 78

3.5c. Effect of Initial Cluster Radius . . . . . . . . . . . . . . . 78

3.5d. Effect of Bubble Volume Fraction . . . . . . . . . . . . . . 80

3.6. Factors Affecting Cavity Cluster Behavior in Acoustic Cavitation . . . . 81

3.7. Concluding Remarks . . . . . . . . . . . . . . . . . . 82

\section{Gas-Liquid Cavitation Chemistry}

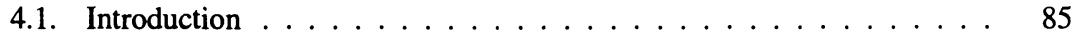

4.2. Mechanisms for Cavitation Reaction . . . . . . . . . . . . . . . . 90

4.3. Factors Affecting Cavitation Chemistry . . . . . . . . . . . . . . . . . . . 96

4.3a. Acoustic Frequency . . . . . . . . . . . . . . . . . . . 96

4.3b. Acoustic Intensity . . . . . . . . . . . . . . . . . . . . . . . . . . . . . . . . . . . . . .

4.3c. External Pressure . . . . . . . . . . . . . . . . . . . . . . . . . . . . . . . . . . . . . . . .

4.3d. Gas Solubility . . . . . . . . . . . . . . . . . . . . . 101

4.3e. Nature of the Gas . . . . . . . . . . . . . . . . . . . . . . . 101

4.3f. Liquid Properties . . . . . . . . . . . . . . . . . . . . . 102

4.3g. Bulk Temperature . . . . . . . . . . . . . . . . . . . . 104

4.4. Inorganic and Organic Cavitation Reactions . . . . . . . . . . . . 106

4.4a. Water . . . . . . . . . . . . . . . . . . . . 107

4.4b. Effect of Other Dissolved Gases . . . . . . . . . . . . . . . . . 110

4.4c. Inorganic Reactions . . . . . . . . . . . . . . . . . . 112

4.4d. Organic Reactions . . . . . . . . . . . . . . . . . . . . 116

4.4e. Solute Hydrophobicity and Reactivity . . . . . . . . . . . . 136 
4.5. Depolymerization and Repolymerization Reactions . . . . . . . . . . . 142

4.6. Ultrasound and Homogeneous Oxidation . . . . . . . . . . . . . . . . 149

4.7. Ultrasound and Liquid-Liquid Phase-Transfer Reactions . . . . . . . . 151

\section{Gas-Liquid-Solid Cavitation Chemistry}

5.1. Introduction . . . . . . . . . . . . . . . . . 155

5.2. General Effects of Ultrasound on Gas-Liquid-Solid Reactions . . . . . 160

5.2a. Surface Cleaning . . . . . . . . . . . . . . . . . . . . 160

5.2b. Morphological Changes in Metal Catalysts . . . . . . . . . . 162

5.2c. Cavitation Erosion . . . . . . . . . . . . . . . . . . . . 162

5.2d. Shape, Size, and Specific Area of Particle . . . . . . . . . . . 163

5.2e. Improved Mass Transport . . . . . . . . . . . . . . . . . . . 163

5.2f. Mechanisms for Gas-Liquid-Solid Cavitation Reaction . . . . . 164

5.3. Specific Role of Ultrasound on Gas-Liquid-Solid Reactions . . . . . . 165

5.3a. Catalyst and Reagent Preparation . . . . . . . . . . . . . 165

5.3b. Effects of Ultrasound on Catalyst-Reagent Activation . . . . . . 172

5.3c. Catalyst Induction Period . . . . . . . . . . . . . . . . . 176

5.3d. Reactions with Continuous Ultrasound . . . . . . . . . . . . . 177

5.3e. Effects of Ultrasound on Catalyst Regeneration . . . . . . . 178

5.4. Case Studies . . . . . . . . . . . . . . . . . . . . . . . . . . . . . . . . 179

5.4a. Cavitation Effect on Heterogeneous Catalytic Oxidation . . . . . 179

5.4b. Cavitation Effect on Liquid-Solid Phase-Transfer Reactions . . 183

5.4c. Cavitation Effect on Gas-Liquid-Solid Biological Reactions . . 185

5.4d. Cavitation Effect on Photo-oxidation Reactions . . . . . . . . 188

5.4e. Cavitation-Induced Microfusion . . . . . . . . . . . . . . . . . . 190

\section{Cavitation Reactors}

6.1. Introduction . . . . . . . . . . . . . . . . . . . . . . 193

6.2. Hydrodynamic Cavitation Reactors . . . . . . . . . . . . . . . . . . . . 194

6.2a. High-Pressure Homogenizer . . . . . . . . . . . . . . . . . . 197

6.3. Acoustic Cavitation Reactors . . . . . . . . . . . . . . . . . . . . . . . 198

6.3a. Transducers and Horns . . . . . . . . . . . . . . . . 201

6.3b. Measurements of Acoustic Power . . . . . . . . . . . . . . . . . 204

6.3c. Methods for Measuring Amplitude . . . . . . . . . . . . . . . 206

6.3d. Hydrophones . . . . . . . . . . . . . . . . . . . . . . 207

6.3e. Sonochemical Reactor Geometries . . . . . . . . . . . . . . 208

6.3f. Qualitative Considerations for Reactor Choice, Scaleup, and

Optimization . . . . . . . . . . . . . . . . 238

6.4. Laser Cavitation Reactors . . . . . . . . . . . . . . . . . . . . . . . . . 240

6.5. Some Additional Considerations for Flow Reactors . . . . . . . . . . . 243

6.6. Health and Safety Aspects of Laboratory Reactors . . . . . . . . . . . . 244

6.7. Integration of Cavitation into Existing Scaled-Up Processes . . . . . . 245

6.8. Concluding Remarks . . . . . . . . . . . . . . . . . . . . 245 


\section{Models for Cavitation Reactors}

7.1. Introduction . . . . . . . . . . . . . . . . . . . 247

7.2. General Considerations for a Gas-Liquid Cavitation Reactor Model . . 248

7.2a. Bubble Dynamics . . . . . . . . . . . . . . . . . . . . . . 249

7.2b. Pyrolysis Reactions in the Bubble . . . . . . . . . . . . . . . 249

7.2c. Free Radical Reactions in the Liquid Film . . . . . . . . . . . 250

7.3. Modeling a Batch Gas-Liquid Acoustic Reactor . . . . . . . . . . . 252

7.3a. Physical Description . . . . . . . . . . . . . . . . . 253

7.3b. Model Equations and Analysis . . . . . . . . . . . . . 254

7.3c. Further Improvements in the Model . . . . . . . . . . . . . . 262

7.4. Characterization of the Reaction Zone . . . . . . . . . . . . . . . 264

7.4a. Physical Description . . . . . . . . . . . . . . . . 265

7.4b. Reaction Zone based on Probability Density Function . . . . . . 267

7.5. Reactor Design and Scaleup based on the Concept of Cavitation Yield . . . 272

7.6. Memory Effect in a Loop Cavitation Reactor . . . . . . . . . . . . . . . 273

7.7. Concluding Remarks . . . . . . . . . . . . . . . . . . 275

8. Energy Efficiency and the Economics of the Cavitation Conversion Process

8.1. Introduction . . . . . . . . . . . . . . . . . . 277

8.2. Efficiency of Energy Transformation . . . . . . . . . . . . . . . . 279

8.2a. Steps for Energy Transformation . . . . . . . . . . . . . . 279

8.2b. Equipment Efficiency . . . . . . . . . . . . . . . . . 280

8.2c. Energy Efficiency for the Cavity Implosion . . . . . . . . . . 280

8.2d. Cavitation Yield Model . . . . . . . . . . . . . . . . . . . 293

8.2e. G-Method for Energy Efficiency . . . . . . . . . . . . . . . . . . 304

8.2f. Case Studies . . . . . . . . . . . . . . . . . . . . . 305

8.3. Economics of Cavitation Conversion Processes . . . . . . . . . . . 307

8.3a. Case Study 1 . . . . . . . . . . . . . . . . . . . 307

8.3b. Case Study $2 \ldots \ldots \ldots \ldots$

8.3c. Sonochemistry vs. Photochemistry . . . . . . . . . 311

8.4. Concluding Remarks . . . . . . . . . . . . . . . . . . . 312

\section{CAV-OX Process}

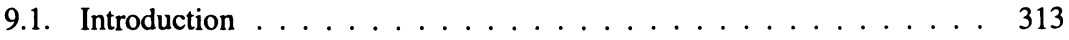

9.2. Description of Process . . . . . . . . . . . . . . . . . . . 313

9.3. Process Economics . . . . . . . . . . . . . . . . . . . . . . . . . . . . 319

9.4. Case Studies . . . . . . . . . . . . . . . . . . . . . . . . 321

Case 1. Superfund Site for Wood-Treatment, Pensacola, Florida . . . . 322

Case 2. Chevron Service Station, Long Beach, California . . . . . . . 323

Case 3. Presidio Army Base, San Francisco, California . . . . . . . 323

Case 4. Chemical Plant, East Coast, United States . . . . . . . . . . 326

Case 5. Mannesmann Anlagenbau, Salzburg, Austria . . . . . . . . . . 327

Case 6. Steel Mill, South Korea . . . . . . . . . . . . . . . . . . . . 327

Case 7. Perdue Farms, Bridgewater, Virginia . . . . . . . . . . . . 328 
Case 8. Southern California Edison, Los Angeles, California . . . . . 330

Case 9. Corporacion Mexicana de Investigacion en Materials, S.A. de C.V. (CMIMSA) . . . . . . . . . . . . . . . . . . 331

Case 10. University of Natal, Durban, South Africa . . . . . . . . . . . 331

Nomenclature $\ldots \ldots \ldots \ldots \ldots \ldots \ldots \ldots \ldots \ldots \ldots$

References . . . . . . . . . . . . . . . . . . . . 339

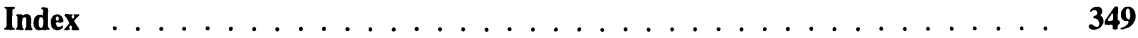




\section{CAVITATION REACTION ENGINEERING}

